\title{
A Unified Characterization of Reproducing Systems Generated by a Finite Family, II
}

\author{
By Eugenio Hernández, Demetrio Labate, and Guido Weiss
}

\begin{abstract}
By a "reproducing" method for $\mathcal{H}=L^{2}\left(\mathbb{R}^{n}\right)$ we mean the use of two countable families $\left\{e_{\alpha}: \alpha \in \mathcal{A}\right\},\left\{f_{\alpha}: \alpha \in \mathcal{A}\right\}$, in $\mathcal{H}$, so that the first "analyzes" a function $h \in \mathcal{H}$ by forming the inner products $\left\{<h, e_{\alpha}>: \alpha \in \mathcal{A}\right\}$, and the second "reconstructs" $h$ from this information: $h=\sum_{\alpha \in \mathcal{A}}$ $<h, e_{\alpha}>f_{\alpha}$.

A variety of such systems have been used successfully in both pure and applied mathematics. They have the following feature in common: they are generated by a single or a finite collection of functions by applying to the generators two countable families of operators that consist of two of the following three actions: dilations, modulations, and translations. The Gabor systems, for example, involve a countable collection of modulations and translations; the affine systems (that produce a variety of wavelets) involve translations and dilations.
\end{abstract}

A considerable amount of research has been conducted in order to characterize those generators of such systems. In this article we establish a result that "unifies" all of these characterizations by means of a relatively simple system of equalities. Such unification has been presented in a work by one of the authors. One of the novelties here is the use of a different approach that provides us with a considerably more general class of such reproducing systems; for example, in the affine case, we need not to restrict the dilation matrices to ones that preserve the integer lattice and are expanding on $\mathbb{R}^{n}$. Another novelty is a detailed analysis, in the case of affine and quasi-affine systems, of the characterizing equations for different kinds of dilation matrices.

\section{Introduction}

The terms reproducing systems or reproducing formulae are applied to any of several methods that "analyze" a vector $v$ (or function) and, then, "reconstructs" $v$ in terms of this analysis. In order to fix our ideas, let us consider a specific way in which this procedure is carried out that will help us explain the principal features of this article.

A countable family $\left\{e_{\alpha}: \alpha \in \mathcal{A}\right\}$ of elements in a separable Hilbert space $\mathcal{H}$ is a frame if

Math Subject Classifications. 42C15, 42C40.

Key Words and Phrases. affine systems, almost periodic functions, Gabor systems, quasi affine systems, tight frames, wavelets.

Acknowledgements and Notes. First author was supported by grants BMF2001-0189 (MCYT) and PR2001-0037

(MECYD); second and third author were supported by a grant from Southwestern Bell. 